\title{
Academic Nursing Center Primary Care Quality Improvement Using an Electronic Health Record and a Practice-Based Research Network
}

\author{
Thomas A. Mackey, Laura Rooney, Delyse Hart \\ School of Nursing, University of Texas Health Science Center at Houston, Houston, Texas, USA \\ Email: Thomas.a.mackey@uth.tmc.edu
}

Received 29 June 2014; revised 24 July 2014; accepted 5 August 2014

Copyright (C) 2014 by authors and Scientific Research Publishing Inc. This work is licensed under the Creative Commons Attribution International License (CC BY). http://creativecommons.org/licenses/by/4.0/

\section{(c) (i) Open Access}

\begin{abstract}
Practice-based research networks (PBRN) seek to improve healthcare through the use of research, quality improvement, and collaborative learning. When used by nontraditional models of care such as the nurse managed healthcare center (NMHC), PBRNs can be incorporated into successful quality improvement (QI) programs. UT Health Services is a NMHC utilizing a PBRN as one component of a comprehensive QI program in an effort to deliver high quality healthcare.
\end{abstract}

\section{Keywords}

Academic Nursing Centers, Practice-Based Research, Nurse Practitioner Clinic Quality Improvement

\section{Introduction}

Nurse Managed Health Center (NMHC) provides an environment for students and faculty to deliver clinical services, perform research and teach students. While some NMHCs offer specialized care to distinctive populations or treat specific health problems, others provide a more general scope of service, most commonly, primary care.

Regardless of the emphasis or objectives of a NMHC, the issue of quality care remains vital. Fourteen specific quality indicator recommendations for NMHCs were outlined by Mackey and McNiel [1] (see Table 1). While such indicators might be a reflection of quality within a NMHC, there were no guarantee evidence-based processes or benchmark outcomes are occurring.

The purposes of the current paper are to review the literature related to NMHCs, quality measurement in 
Table 1. Quality indicator recommendations for a nurse managed health center [1].

- Quality assurance program

- Financial stability

- Educational opportunities for students

- $\quad$ Research efforts

- $\quad$ Patient care processes

- $\quad$ Billing \& insurance systems

- Administration \& governance

- Marketing efforts

- Clinical records \& continuing education for faculty \& staff

- $\quad$ Credentialing \& continuing education for faculty \& staff

- $\quad$ Clean \& safe environment/facilities

- Health education \& wellness services

- $\quad$ Employee policies \& procedures

primary care and practice-based research networks (PBRN) and then discuss the quality improvement (QI) program of a particular NMHC utilizing a selected electronic health record (EHR) and participating in a PBRN.

\section{Review of Literature}

\subsection{Nurse Managed Healthcare Centers (NMHC)}

A number of definitions exist for NMHCs. Among the definitions is the following: "A nurse managed health center is an accessible service that delivers family and community oriented primary health care. The majority of care is provided by nurse practitioners in collaboration with other nursing and other health care providers, e.g. social workers, physicians, dentists” [2].

The development of the modern NMHC evolved from early models of community based nursing care which focused on the needs of communities and families [1]-[3]. Although meeting the needs of communities and families has remained a core principle in NMHCs, the demand for affordable and accessible healthcare coupled with expanding practice roles for nurse practitioners, has allowed the transition to today's model of care in which nurses occupy the chief management position and have accountability and responsibility for client care as primary providers [1].

Transitioning from the community health care nurse model of 1893 to the modern version of the NMHC with an advanced practice nurse as primary care provider has been a path fraught with challenges and lessons learned. Leaders from the National Nursing Centers Consortium [4] identify the 1960s as pivotal in the development of today's model [5]. During the 1960's federal and state laws identified the provision of healthcare to the underserved as an unmet need, with the holistic model of nursing care poised to fill the healthcare gap. However, not until the 1980s and 1990s, when grants provided by the U.S. Health Services and Resources Administration (HRSA) were to be used for the establishment of NMHCs, did the number begin to exponentially grow. The typical NMHC was aligned with an academic institution with goals of providing care and services to underserved communities, while serving as a clinical site for both faculty and students [3]. Many of the initial programs struggled to maintain financial viability once grant funding ended. Due to financial loses, some centers relied on institutional subsidies and struggled for years before closing [3]. Despite the financial shortfalls of the NMHC model, today there are approximately 250 nurse-managed clinics. Seventy-four percent are affiliated with a school of nursing and $26 \%$ are independent not-for-profit or connected with a hospital outpatient department [6].

Throughout the history of NMHCs mentioned above there has been little emphasis on quality of care. However, such days of inattention to quality have ended. Additionally, the advent of the Affordable Care Act complicates how NMHCs will manage and develop infrastructure in order to survive. Nonetheless, quality of care will remain of paramount importance as nurse practitioners strive to be part of the solution to the health care issues in the United States.

\subsection{Quality Measures: Use of HEDIS Guidelines in Practice}

NMHCs account for approximately 2.5 million patient encounters annually with a capacity for more, uniquely 
positioning them as a solution to the shortage of primary care providers [6]. However, the use of nurse practitioners as independent healthcare providers has met with opposition and quality of care is one of the issues in the debate. Despite the fact that research has demonstrated advanced practice nurses deliver safe and effective care, use of a standardized measure of performance would leave little room for continued debate [7] [8].

One such standardized measure of quality performance is the Healthcare Effectiveness and Data Information Set (HEDIS). HEDIS is a nationally recognized performance measurement tool utilized by $90 \%$ of healthcare plans and clinical settings to compare performance [4]. Developed by the National Committee on Quality Assurance (NCQA), HEDIS consists of approximately 74 performance measures over five domains of care and service. Measures of outcomes, overuse, resource use, and care coordination are included within the data set and NCQA strives to provide meaningful, practical, and valuable information in reporting HEDIS measures. The process for evaluating quality measures is rigorous and continuously evaluated for effectiveness and utility. As a result, HEDIS is one of the most widely used measurement tools of quality performance in ambulatory care.

There is minimal published research regarding the use of HEDIS measures in NMHCs, indicating a need for more. However, Barkauskas et al. [9] offer an explanation as to why the use of HEDIS in NMHCs is challenging, stating that most NMHCs do not have the high patient volume or managed care arrangements characteristic of other types of primary care practices. Additionally, most NMHCs provide more episodic are versus management of chronic health conditions. Nevertheless, HEDIS measures are important in the evaluation of primary care practices. Additionally, the Centers for Medicaid and Medicare (CMS) have used similar measures in the development of their physician quality initiative (PQRI) pay for performance plan. Under the PQRI plan, providers are offered financial incentives to reach outcome goals as established in the HEDIS measures.

\subsection{Practice-Based Research Networks}

The history of practice-based research networks (PBRN) dates back to the 1970s and 1980s when the Agency for Healthcare Research and Quality (AHRQ) began to establish networks of clinicians who not only practiced, but were also committed to engaging in collaborative research relevant to their practices in order to address issues of quality and evidence-based practice [10]-[12]. Initially comprised of physician-owned practices, the collaborative networks were funded and supported by the National Institutes of Health (NIH) in order to speed the translation of research into practice. There are approximately 111 networks within the United States, most in primary care, and the networks are an effective tool for primary care providers seeking to practice evidence based medicine [10]-[13].

PBRNs are a unique tool in the advancement of healthcare, and particularly primary care. Practice inquiries answered through the collaborative networks generally are not addressed through a traditional form of research. As a result, the outcomes data are invaluable to practicing clinicians who need answers to everyday clinical challenges [14]. Health disparities, health prevention and promotion, diabetes, hypertension, and mental health are but a few examples that demonstrate how PBRNs are used in the clinical setting [14]. PBRNs are well represented in the literature, contributing over 600 peer-reviewed articles [14].

PBRNs, otherwise referred to as collaborative learning environments, use traditional and nontraditional methods to improve primary care processes and patient outcomes [15]. An example of a PBRN is thePractice Partner Research Network (PPRNet), a network whose members utilize the same electronic health record. PPRNet seeks to advance the healthcare delivered by its participants through the use of research, quality improvement, and collaborative learning [15]. Although originally comprised solely of physician-owned practices, PPRNet is open to clinics using McKesson Practice Partner as an EHR, which includes NMHCs. Recognized as a critical component in improving translational research, participation in PBRNs allows for a review and comparison of current practice to a national standard in order to improve care delivery for improved patient outcomes.

The literature is limited regarding specific quality outcomes and improvement recommendations for NMHCs utilizing an EHR within a PBRN. However, one study in particular did address NMHCs and PBRN. Barkauskas [16] discovered the overall, quality measure findings for breast cancer screening, cervical cancer screening, diabetes care, hypertension management, and smoking cessation compared favorably with national benchmarks, with particularly high quality demonstrated for chronic disease care management. The nine participating clinics submitted a combination of manual and electronic data for analysis and representing a significant attempt to measure outcomes with an EHR in a PBRN. Overall, the centers reached the $50^{\text {th }}$ percentile for Healthcare Effectiveness Data and Information Set (HEDIS) measures of the above data points and the $90^{\text {th }}$ percentile bench- 
mark for most of the quality measures.

\section{UTHS and Practice Partner Research Network (PPRNet)}

The University of Texas Health Services (UTHS) clinic opened for service in February, 1990 with the mission of being a model academic health services center for the $21^{\text {st }}$ century in the areas of patient care, education and research. UTHS provides comprehensive, efficient, effective and high quality health care services valued by clients. Key services are primary care, occupational health, travel medicine, and diabetes education. UTHS has some 10 - 12,000 patient visits per year, operates on a fiscally sound basis and provides student learning experiences.

In early 1994 the UTHS adopted an integrated EHR called Practice Partner providing patient record keeping, appointment scheduling and billing services. Throughout the years, Practice Partner evolved from a DOS based computer program to a fully integrated nationally certified EHR compatible with the requirements to become a Certified Medical Home.

Features of PPRNet include quarterly extraction of de-identified patientdemographic information, diagnosis, medications, laboratory results, and vital signs. Progress notes, consultation reports, and discharge summaries are not included. Quarterly reports show performance on 83 (see Table 2) care indicators and compare the UTHS practice providers within the network to national benchmarks. Benchmarks are derived from published evidence based sources, such as the CDC, NIH and AHRQ and Healthy People 2010, NCQA Health Plan Employer Data and Information Set (HEDIS) and the AHRQ National Healthcare Quality Report (NHQR).

Table 2. University of Texas health services quality measures reported on quarterly basis.

\section{Diabetes Mellitus}

1. DM patients with blood pressure measured in past 6 months

2. DM patients with most recent blood pressure $<140 / 90$

3. DM patients with glycosylated hemoglobin measured in past 6 months

4. DM patients with most recent glycosylated hemoglobin measurement $<7 \%$

5. DM patients with most recent glycosylated hemoglobin measurement $<8 \%$

6. DM patients with LDL-cholesterol measured in the past year

7. DM patients with most recent LDL-C $<100 \mathrm{mg} / \mathrm{dl}$

8. DM patients with urinary microalbumin measured in past year

Cardiovascular Disease

9. Patients $\geq 18$ years with BP measured in past 2 years

10. Patients diagnosed with HTN for 3 BP measures $\geq 140 / 90$ in past year

11. HTN patients with BP measured in past 6 months

12. HTN patients with most recent BP $<140 / 90$ in past 6 months

13. HTN patients with blood glucose in the past 3 years

14. Men $\geq 35$ and women $\geq 45$ screened for cholesterol in the past 5 years

15 . Men $\geq 35$ and women $\geq 45$ HDL-C screen measure in the past 5 years

16. CHD or atherosclerosis patients with LDL-cholesterol measured in past year

17. CHD or atherosclerosis patients with most recent LDL-C $<100 \mathrm{mg} / \mathrm{dl}$

18. CHD or atherosclerosis patients with current lipid lowering $\mathrm{Rx}$

19. High Risk patients with current anti-platelet Rx

20. Patients with atrial fibrillation with current anti-platelet or oral anticoagulant Rx

21. Patients with DM and HTN with current ACE inhibitor or ARB Rx

22. Patients with systolic heart failure with current ACE inhibitor or ARB Rx

23. Patients with systolic heart failure with current Beta blocker Rx

24. Patients screened for abdominal aortic aneurysm 


\section{Continnued}

\section{Women's Health Care}

25. Screening for genital Chlamydia in last year in 16 - 24 year-old women

26. Women with bone density ever measured since age 65

\section{Cancer Screening}

27. Women 21 - 64 years without hysterectomies with Pap test in past 3 years

28. Women 50 - 74 years with Mammogram in past 2 years

29. Patients 50 - 75 years up to date for colorectal cancer screening

Immunizations
30. Patients $\geq 12$ years with Tetanus vaccination in past 10 years
31. Patients $\geq 6$ months with Influenza vaccination in past year
32. Patients $\geq 65$ years with Pneumococcal vaccination ever
33. High Risk patients 18 - 64 years with Pneumococcal vaccination ever
34. Patients with liver disease with two Hepatitis A vaccinations ever
35. Patients 11 - 19 years with Meningococcal vaccination ever
36. Women 9 - 26 years old with three HPV vaccinations ever
37. Patients $\geq 60$ years with Zoster (shingles) vaccination ever

Mental Health and Substance Abuse
38. Patients screened for depression in past 2 years
39. Patients with depression with current anti-depressant $\mathrm{Rx}$
40. Patients screened for alcohol use in past 2 years
41. Patients with alcohol diagnosis or at-risk drinking with alcohol counseling in past year
42. Patients with alcohol diagnosis with current alcohol prescription
43. Patients $\geq 13$ years screened for tobacco use in past 2 years
44. Current tobacco use patients with smoking intervention in past year

\section{Respiratory Disease}

45. Asthma patients $\geq 5$ years with current controller $\mathrm{Rx}$

46. Patients without antibiotic Rx within 3 days of Dx of URI/pharyngitis/bronchitis

47. Patients with sinusitis/strep pharyngitis/otitis media/COPD exacerbation prescribed antibiotics with narrow spectrum antibiotic Rx

\section{Medication Safety}

48. Avoiding potentially inappropriate medications in patients $\geq 65$ years

49. Avoiding rarely appropriate medications in patients $\geq 65$ years

50. Appropriate dosages of Benzodiazepines in patients $\geq 65$ years

51. Appropriate dosages of $\mathrm{H} 2$ blockers in patients with $\mathrm{CrCl}<50 \mathrm{ml} / \mathrm{min}$

52. Avoid Rx of anticholinergic in patients with Dx of dementia

53. Avoid Rx for NSAID or Cox 2 Inhibitor in patients with Dx of Heart Failure

54. Cautious use of NSAID or Cox 2 Inhibitor in patients with Dx of Hypertension

55. Avoid Rx for Thiazolidinedione in patients with Dx of Heart Failure

56. Avoid Rx for Metformin in patients with most recent Serum Creatinine in past year (men with SCr $\geq 1.5 \mathrm{mg} / \mathrm{dl}$ and women with $\mathrm{SCr} \geq 1.4 \mathrm{mg} / \mathrm{dl})$

57. Serum creatinine measured in past year in patients with Rx for any ACE Inhibitor or A II Inhibitor, Digoxin, any diuretic, or Metformin

58. Serum creatinine measured in past six months in either (patients $\geq 65$ years or with $\mathrm{CrCl}<50 \mathrm{ml} / \mathrm{min}$ ) and $\mathrm{Rx}$ for (ACE Inhibitor or A II Inhibitor) and K Sparing diuretic

59. Most recent Potassium measurement $\geq 3.5 \mathrm{meq} / \mathrm{L}$ in patients with $\mathrm{Rx}$ for any thiazide and Potassium measure in past year

60. Hemoglobin measured in past year in patients with Rx for any Anti-Platelet (excluding Aspirin) or Oral Anticoagulant

61. Glucose measured in past year in patients with Rx for any Antipsychotic

62. Patients with active Rx for Warfarin with INR measured in past 45 days 
The purposes of the quality improvement (QI) program at UTHS are:

- to measure the safety, efficiency and quality of care delivered to patients;

- to improve delivery of care to patients;

- to provide data to health care providers regarding care processes and outcomes needed for changing care processes.

QI at UTHS involves participation with PPRNet, managed care and insurance company reviews, university audits including coding, financial, and administrative reviews.

As follow up to feedback from internal and external reports, staff meetings are held on a weekly basis addressing business, staffing and quality of care matters. Nurse practitioners have initiated specific QI projects directed toward improvement of care for patients with chronic diseases such as diabetes [17] and hypertension to adherence to the US Preventive Task Force recommendations for health maintenance.

Quality of care is defined differently by patients, providers, administrators. While patients may judge the quality of services received in terms of satisfaction, providers are more concerned with processes and particularly, outcomes of care. Quality is the degree of excellence by which a service is rendered and measured against a standard. Standards are established by individuals, governments or, in the case of health care, nation$\mathrm{al} /$ international professional organizations and associations. Systematically improving the degree of excellence, based on benchmarks or standards, is the meaning of a quality improvement program.

Satisfaction is a patient's subjective perception regarding treatment received. If a patient approves or agrees with the experience then approval will be high. If approval is low, satisfaction will be low and the quality of care is perceived as low.

Process is a method, manner or means by which a course of action is performed. Of note, however, there is a lack of strong scientific evidence between processes and meaningful outcomes [18].

Outcome is the result, effect, conclusion or end effect of a process.

Quality indicator is a policy, program, protocol, standard, guideline, assessment measure, or other evaluation tool that shows there is reason to believe measures are in place to assure a high level of care is provided [1].

\section{Implications for Other Nurse Practitioner Practices Using an EHR}

Discussions of how to successfully reengineer the way healthcare is delivered in the United States continue to be a topic of debate. In 2002, the landmark, Institute of Medicine (IOM) report, To Err is Human, brought to light the enormous numbers of preventable medical errors and the economic burden it placed on an already overwhelmed healthcare system. Quality or the lack thereof, becomes the central theme in every healthcare discussion, and continues as one of the major talking points today.

A later IOM study reported on the strengths and weaknesses of our healthcare system, how to streamline and cut down on medical errors and waste, and addressed the issues of supply and demand utilizing the current workforce. Nurse practitioners were identified as a valuable, yet underutilized, resource that would easily fill the workforce void. Individual states, however, should facilitate with legislation for NPs. To date only 13 states have practice laws favorable to NP practice. With the prospect of millions of new participants in the healthcare system as the Affordable Care Act takes place in 2014, the NP quality issue needs to be answered once and for all to take advantage of an underused resource.

PBRNs, directly linked to quality and patient outcomes, can answer the quality debate. By comparing similar size practices and performance on identified quality measures, PBRNs level the playing field. In the PBRN, NPs are directly compared to providers of similar skill set, including MDs and NPs. Outcomes are measured, compared, and reported. For the NMHC, PBRNs could demonstrate, on a large scale, NPs produce the same quality patient outcomes as traditional physician-led Practices. National standards drive the successful practices, and as such, best practice both within and amongst practices can be identified. PBRNs help to facilitate CQI programs, addressing deficiencies quickly, further enhancing quality and improving patient outcomes in today's rapidly changing healthcare environment. With an ever-aging and more chronically ill population, PBRNs can deliver information that providers need to formulate clinical decisions, and then evaluate the outcomes to improve care. PBRNs allow providers to raise the question: “Am I doing the best I can do for my patients?”

\section{Funding}

No funding was received for this project and no author has any conflict of interest. 


\section{References}

[1] Mackey, T.A. and McNiel, N.O. (2002) Quality Indicators for Academic Nursing Primary Care Centers. Nursing Economics, 20, 62-73.

[2] Institute for Nursing Centers (2012)

http://www.nursingcenters.org/PDFs/NMHC\%20Brochure\%20INClayoutFINAL.pdf

[3] King, E.S. (2007) A 10-Year Review of Four Academic Nurse-Managed Centers: Challenges and Survival Strategies. Journal of Professional Nursing, 24, 14-20. http://dx.doi.org/10.1016/j.profnurs.2007.04.003

[4] National Committee on Quality Assurance (2012) Continuous Improvement and the Expansion of Quality Management. Accessed January 28. http://www.ncqa.org/LinkClick.aspx?fileticket=FpMqqpADPo8\%3d\&tabid=836

[5] Nurse-Managed Health Centers (2012) Assuring Quality Care. National Nursing Centers Consortium Quality Task Force Report. Available 21 January 2012. www.nncc.us/site/pdf/NMHC_Quality_Standards.pdf

[6] Hansen-Turton, T., Bailey, D.N., Torres, N. and Ritter, A. (2010) Nurse-Managed health Centers: Key to a Healthy Future. American Journal of Nursing, 110, 23-26. http://dx.doi.org/10.1097/01.NAJ.0000388257.41804.41

[7] Mundinger, M.O., Kane, R.L., Lenz, E.R., Totten, A.M., Tsai, W.Y., Cleary, P.D., et al. (2000) Primary Care Outcomes in Patients Treated by Nurse Practitioners or Physicians: A Randomized Trial. JAMA, 283, 59-68. http://dx.doi.org/10.1001/jama.283.1.59

[8] Horrocks, S., Anderson, E. and Salisbury, C. (2002) Systematic Review of Whether Nurse Practitioners Working in Primary Care Can Provide Equivalent Care to Doctors. British Medical Journal, 324, 819-823. http://dx.doi.org/10.1136/bmj.324.7341.819

[9] Barkauskas, V.H., Pohl, J.M., Benkert, R. and Wells, M.A. (2005) Measuring Quality in Nurse-Managed Centers Using HEDIS Measures. Journal for Healthcare Quality, 27, 4-14. http://dx.doi.org/10.1111/j.1945-1474.2005.tb00540.x

[10] Fagnan, L.J., Davis, M., Deyo, R.A., Werner, J.J. and Stange, K.C. (2010) Linking Practice-Based Research Networks and Clinical and Translational Science Awards: New Opportunities for Community Engagement by Academic Health Centers. Academic Medicine, 85, 476-483. http://dx.doi.org/10.1097/ACM.0b013e3181cd2ed3

[11] Deshefy-Longhi, T., Swartz, M.K. and Grey, M. (2007) Characterizing Nurse Practitioner Practice by Sampling Patient Encounters: An APRNet Study. Journal of the American Academy of Nurse Practitioners, 20, 281-287. http://dx.doi.org/10.1111/j.1745-7599.2008.00318.x

[12] Green, L.A. and Hickner, J. (2005) A Short History of Primary Care Practice-Based Research Networks: From Concept to Essential Research Laboratories. Journal of the American Board of Family Medicine, 19, 1-10. http://dx.doi.org/10.3122/jabfm.19.1.1

[13] Tierney, W.M., Oppenheimer, C.C., Hudson, B.L., Benz, J., Finn, A., Hickner, J.M, et al. (2007) A National Survey of Primary Care Practice-Based Research Networks. Annals of Family Medicine, 5, 242-250. http://dx.doi.org/10.1370/afm.699

[14] Tapp, H. and Dulin, M. (2010) The Science of Primary Health-Care Improvement: Potential and Use of Community-Based Participatory Research by Practice-Based Research Networks for Translation of Research into Practice. Society for Experimental Biology and Medicine, 235, 290-299. http://dx.doi.org/10.1258/ebm.2009.009265

[15] Mold, J.W. and Peterson, K.A. (2005) Primary Care Practice-Based Research Networks: Working at the Interface between Research and Quality Improvement. Annals of Family Medicine, 3, S12-S20. http://dx.doi.org/10.1370/afm.303

[16] Barkauskas, V.H., Pohl, J.M., Tanner, C., Onifade, T. and Pilon, B. (2011) Quality of Care in Nurse-Managed Centers. Nursing Administration Quarterly, 35, 34-43. http://dx.doi.org/10.1097/NAQ.0b013e3182032165

[17] Mackey, T., Cole, F. and Lindenberg, J. (2005) Quality Improvement and Changes in Diabetic Patient Outcomes in an Academic Nurse Practitioner Primary Care Practice. Journal of the American Academy of Nurse Practitioners, 17, 547-553. http://dx.doi.org/10.1111/j.1745-7599.2005.00090.x

[18] Schillie, S. (2012) Quality Improvement in Healthcare. Accessed on 2/23/12. http://www.medscape.org/viewarticle/561651 
Scientific Research Publishing (SCIRP) is one of the largest Open Access journal publishers. It is currently publishing more than 200 open access, online, peer-reviewed journals covering a wide range of academic disciplines. SCIRP serves the worldwide academic communities and contributes to the progress and application of science with its publication.

Other selected journals from SCIRP are listed as below. Submit your manuscript to us via either submit@scirp.org or Online Submission Portal.
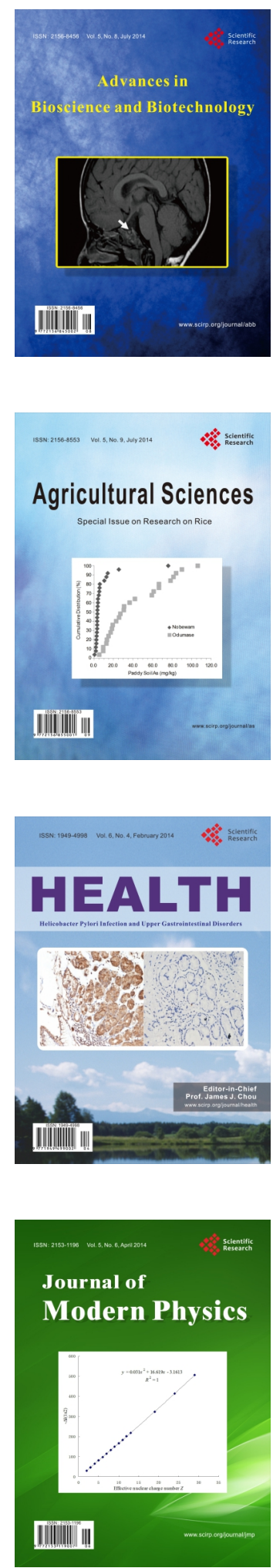
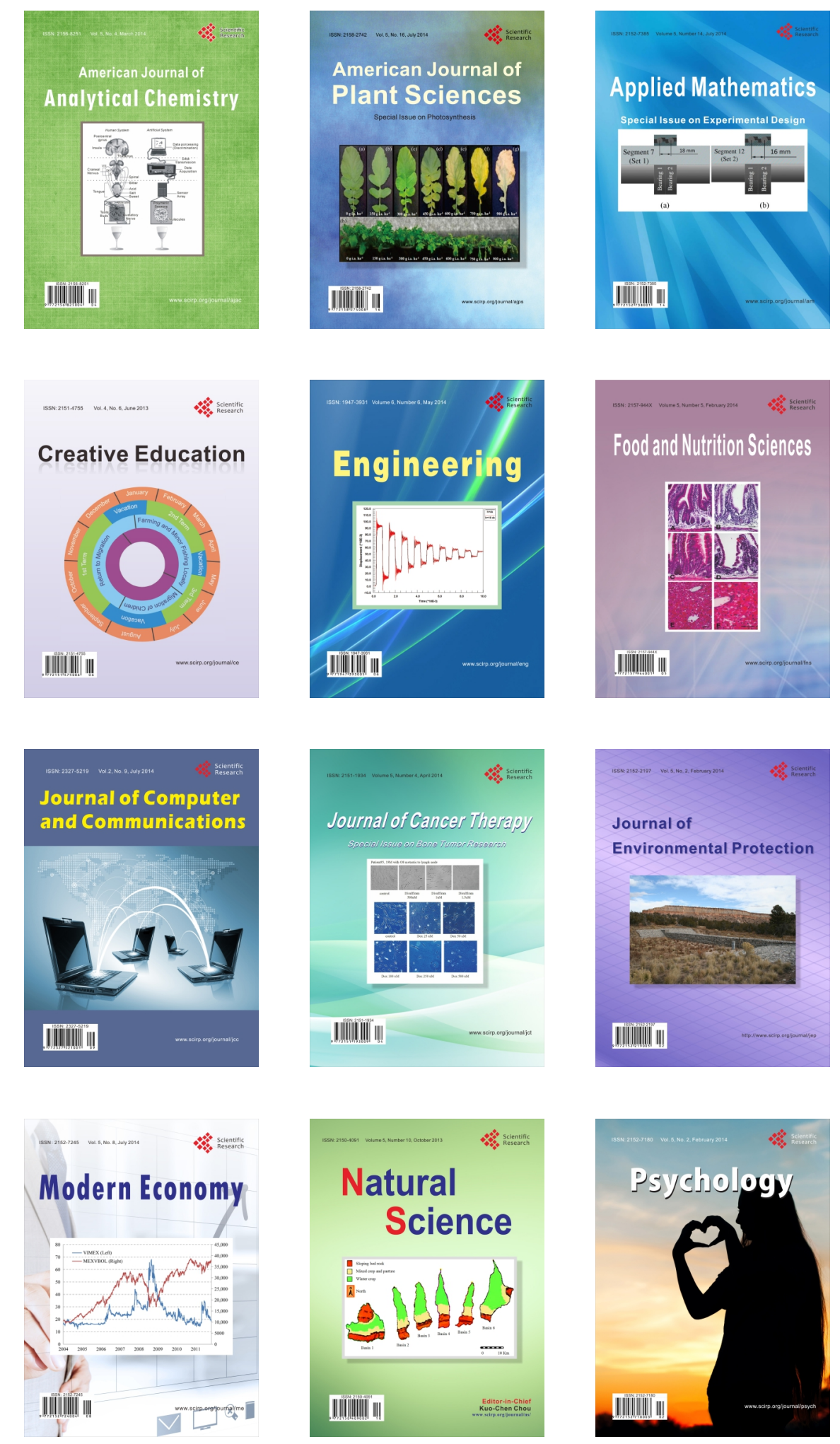